\title{
Les partenariats école-communauté dans les écoles de langue française en Ontario ${ }^{1}$
}

\author{
Nathalie Bélanger et Geneviève Beaulieu \\ Université d'Ottawa
}

\section{Résumé}

Les partenariats entre des acteurs des écoles de langue française et ceux du milieu communautaire et associatif sont de plus en plus courants en Ontario. Or, on ne sait que très peu à leur sujet. Cet article tente de mieux éclairer ce que signifie l'entreprise partenariale pour les acteurs scolaires. À partir d'une recension des écrits, une définition idéaltypique d'un partenariat école-communauté est construite et quatre dimensions sont identifiées pour guider une analyse plus approfondie des partenariats : l'identification et la provenance des partenaires (secteurs scolaire et/ou communautaire), l'initiative du projet, sa durée et les rôles dévolus à chacun des partenaires. L'analyse des résultats de sondages auprès des directions des conseils scolaires et de questionnaires auprès de directions scolaires démontre que les écoles de langue française en Ontario entretiennent avec leur communauté des activités diversifiées surtout pour le bénéfice des élèves. L'analyse dévoile par contre qu'une certaine confusion règne quant à la définition d'un partenariat école-communauté chez les acteurs scolaires interrogés. Même si plusieurs d'entre eux considèrent leurs projets ponctuels comme des partenariats école-communauté, peu de partenariats déclarés se rapprochent de la définition idealtypique élaborée dans le cadre de cette étude.

\begin{abstract}
Partnerships between French language schools and their community actors are more and more common in Ontario. However, we know very little about them. This article aims to understand what partnerships mean to school actors. Based on a literature review, an ideal-typical definition is constructed and four characteristics are identified to guide further analysis of partnerships : the partners' identification and origin (school or community sectors), the project's initiative, its length and the roles assigned to each partner. The results of surveys to the school board's directions and questionnaires to school's principals show that a variety of activities, mainly for the benefits of students, exist between French language schools in Ontario and their community. The analysis reveals, however, that many of the participant school actors are uncertain about the definition of a school-community partnership. Many of them consider their specific one-shot projects as school-community partnership. In fact, few of the declared partnerships come close to the ideal-typical definition formulated in this article.
\end{abstract}

\footnotetext{
${ }^{1}$ Cette étude découle d'un projet de recherche plus vaste commandé et financé par le ministère de l'Éducation de l'Ontario. Nous aimerions remercier les coauteures de cette étude, Phyllis Dalley et Liliane Dionne ainsi que Tina Desabrais, Anne-Sophie RuestPaquette et Hélène Leone qui nous ont assistées lors de la cueillette des données.
} 


\section{Introduction}

Les écoles, et particulièrement celles en milieu minoritaire, sont encouragées à tisser des liens étroits avec les divers partenaires de leur communauté (Association canadienne d'éducation de langue française-ACELF, 2006; Dubé, 1999; Fédération culturelle canadienne-française-FCCF, 2006; Gérin-Lajoie, 2000; Ministère de l'Éducation de I'Ontario-MÉO, 2004; Ouellette et LeBreton Forbes, 1995). En Ontario, le ministère de l'Éducation, dans sa politique d'aménagement linguistique, incite de plus en plus les écoles et les communautés francophones à établir des partenariats dans divers domaines : santé, services sociaux et communautaires, petite enfance, etc. (MÉO, 2004). Les avantages que retirent les écoles et les communautés engagées dans des partenariats ont abondamment été documentés (Barbour et Barbour, 2001; Bouillion et Gomez, 2001; Burke et Picus, 2001; Lewis, 1999; Navarro et Natalicio, 1999; Scales, Roehlkepartain, Neal, Kielsmeier et Benson, 2006; Schweisfurth, 2005; Shatkin et Gershberg, 2007). Entre autres, il semble que les partenariats entre des acteurs scolaires et communautaires en situation minoritaire permettent d'assurer le recrutement et la rétention des élèves (FCCF, 2006; Gérin-Lajoie, 2000; Rainville, 2001), tout en contribuant à la construction ou reconstruction socioéconomique des communautés francophones plus isolées des grands centres urbains et, par conséquent, privées de ressources pouvant favoriser leur développement et leur émancipation (Bélanger, Dalley, Dionne et Beaulieu, 2007a). Les entreprises partenariales permettent même aux écoles de s'assurer d'une certaine visibilité dans un marché scolaire de plus en plus compétitif (Bélanger, Dalley, Dionne et Beaulieu, soumis).

L'intérêt grandissant envers les partenariats écoles-communautés nous amène à nous questionner sur la nature de ceux-ci, à savoir quelle signification les acteurs scolaires accordent à ce type d'entreprises, quelles sont les pratiques qui leur sont associées, afin, éventuellement, de mieux outiller les écoles dans l'établissement de partenariats plus solides et utiles. 
À partir d'une enquête menée en 2006 et 2007, nous dressons ici un portrait des projets de partenariat qui existent entre des acteurs scolaires des écoles de langue française en Ontario et ceux du milieu communautaire et associatif. La communauté est entendue comme regroupant tout acteur individuel ou collectif gravitant autour de l'école : il peut s'agir de parents, d'organismes publics, parapublics ou privés. Nous tentons de mieux comprendre ce que signifie l'entreprise partenariale entre les écoles de langue française en situation minoritaire en Ontario et leur communauté plus large, dans un contexte où la mise en œuvre de tels partenariats est de plus en plus encouragée. Ainsi, dans un premier temps, il convient d'élaborer, à partir de la recension des écrits, une définition de la notion de partenariat qui permette de mieux situer et analyser les partenariats déclarés et recensés. Par la suite, la méthodologie utilisée pour l'enquête sur le terrain est présentée. L'analyse des données issues de sondages et de questionnaires permet de préciser la signification que les acteurs scolaires accordent à leurs entreprises partenariales.

\section{Élaboration d'une définition idéaltypique d'un partenariat école-communauté}

Afin de cerner les éventuels éléments constitutifs d'une définition idéaltypique du partenariat, nous avons effectué une recherche dans la littérature internationale portant sur les relations entre les écoles et les communautés. Nous avons dirigé notre regard plus spécifiquement sur les situations minoritaires dans le cadre d'une recherche dans Internet, dans Orbis, le catalogue de l'Université d'Ottawa, et dans des banques de données contenant des références dans les domaines de l'éducation et des sciences humaines et sociales, à partir d'une combinaison de mots-clés tels que school*, communit*, francophon*, partnership*, minorit*. Une soixantaine de textes ont été recensés et consignés à l'aide du logiciel FileMaker Pro. L'analyse de ces textes a permis de faire ressortir, d'une part, les conditions de mise en œuvre et les retombées positives des entreprises partenariales et, d'autre part, les limites et les difficultés recensées par les divers auteurs.

Le partenariat renvoie, selon son acception la plus commune, à une forme de coopération entre deux ou plusieurs organisations concourant à réaliser un projet par la mise en commun de moyens matériels, intellectuels, humains ou financiers. Les partenariats école-communauté renvoient à des collaborations entre des écoles et des individus, des entreprises, des institutions ou organismes, formels ou informels, dans une communauté (Sanders, 2003). Selon Epstein et Sanders (2000), en plus de l'école et de la famille, la communauté englobe les commerces, les organismes, les quartiers, les cercles d'amitié et autres associations. Les lois, règlements, ressources, services et programmes communautaires peuvent diluer ou renforcer les liens entre ces groupes. Newman (2001) établit une distinction entre « réseaux » (networks) et «partenariats » (partnerships) : « Networks are informal and fluid, with shifting membership and ambiguous relationships and accountabilities » [...] Partnerships « are more stable groupings with defined structures and protocols » (p. 108). Les partenariats, d'après lui, ont une visée plus large et structurée que les réseaux. Ball (2007) abonde dans le même sens en définissant les partenariats comme des unités ou regroupements stables engageant la participation d'une variété d'organismes et d'acteurs sociaux, et possédant des structures et des modes de fonctionnement bien définis.

Dans leur analyse des partenariats école-communauté pour le succès scolaire des élèves de minorités linguistiques aux États-Unis, Adger et Locke (2000) concluent qu'afin d'assurer l'engagement complet des partenaires potentiels, il est nécessaire d'élaborer une vision claire et commune du partenariat, dans laquelle les responsabilités de chaque partenaire sont précisées et ce, dès les débuts de la collaboration. Les partenaires s'engagent afin de 
réaliser des buts communs et de bénéficier de retombées positives ou d'avantages mutuels (Bouillion et Gomez, 2001). En outre, il apparaît que les partenariats sont souvent amorcés dans le but de s'attaquer à certains problèmes ou de répondre à des situations jugées fragiles que vit, à titre d'exemple, une population d'élèves à risque de marginalisation ou d'exclusion (Broccolichi et van Zanten, 1997; Epstein et Sanders, 2000; Lewis, 1999; Navarro et Natalicio, 1999; van Zanten, 1997). De plus, les partenariats école-communauté requièrent des aptitudes à collaborer de la part de ceux et celles qui s'y engagent, l'établissement de rôles précis et l'adoption d'un processus décisionnel partagé entre les partenaires (Sanders, 2003). Les partenariats exigent, de surcroît, une démarche réflexive et évaluative (Sanders, 2003). En situation minoritaire, les partenaires sont amenés à définir leur conception de la communauté et le but de l'entreprise partenariale (Bélanger, Dalley, Dionne et Beaulieu, 2007a, 2007b).

Il existe diverses façons de catégoriser les partenariats école-communauté : en fonction du domaine d'activités concerné (culturel, social, économique ou international); en fonction des partenaires engagés (partenariat interne ou intrascolaire, dans un établissement, entre établissements de même niveau, interniveaux ou externe); selon les modes de fonctionnement (fonctions remplies par les acteurs et relations établies entre eux) (Zay, 2005). Les partenariats peuvent aussi être catégorisés en fonction de leurs objectifs, qu'il s'agisse d'activités centrées ou axées sur l'élève, sur la famille, sur l'école ou sur la communauté (Sanders, 2003). Les activités centrées sur l'élève fournissent des biens et services à l'élève tels que, par exemple : bourses (Warren, 2005), tutorat (Adger et Locke, 2000), orientation de carrière (Chen, Konantz, Rosenfeld et Frost, 2000). Les activités centrées sur l'école procurent des avantages à l'école : embellissement de l'école (Evergreen, 2008), ressources financières et matérielles pour l'école (Gérin-Lajoie, 1995), développement professionnel des enseignants (Lewis, 1999; Navarro et Natalicio, 1999; Warren, 2005). Les activités centrées sur les familles visent à soutenir celles-ci ou, plus spécifiquement, les parents : ateliers pour les parents (Bouillion et Gomez, 2001; Farmer et Labrie, 2008; Warren, 2005), counselling familial (Barbour et Barbour, 2001). Enfin, les activités centrées sur la communauté s'orientent vers l'amélioration des conditions de vie de celle-ci et de ses membres: activités charitables (Warren, 2005), expositions artistiques et scientifiques (FCCF, 2006; Thomson, 2006), revitalisation de la communauté (Ouellette et Lebreton Forbes, 1995) et projets d'embellissement de la communauté (De Almeida, 2003). En situation minoritaire, les partenariats centrés sur les élèves, l'école, la famille et la communauté plus large ont souvent pour but de rendre plus visible l'école dans sa communauté (Bélanger, Dalley, Dionne et Beaulieu, sous presse).

Or, si les partenariats école-communauté constituent des entreprises avantageuses et profitables à bien des égards, ils ne sont pas sans risques. En effet, les partenariats sont quelquefois mis à l'épreuve par des difficultés de fonctionnement. Les acteurs peuvent rencontrer des problèmes lorsque leurs initiatives se heurtent à des réseaux plus larges et mieux établis dans le paysage sociopolitique (Paglin, 1998). En particulier, lorsque les partenaires proviennent majoritairement du monde de l'éducation, il leur est plus difficile de saisir les enjeux associés aux relations sociales et aux relations de pouvoir que leurs écoles entretiennent dans le contexte sociopolitique plus large, notamment en ce qui concerne les questions de statuts, ou les circonstances historiques et socio-économiques qui jouent un rôle dans le façonnement de la communauté (Leistyna, 2002).

Des conflits dans la gestion des partenariats reliés, par exemple, au peu de stabilité des partenaires engagés (Barbour et Barbour, 2001) ou à la présence de styles de leadership et d'engagement peu complémentaires, risquent 
d'engendrer une perte d'orientation du projet et de rendre difficile sa bonne marche (Paglin, 1998). Edens et Gilsinan (2005) notent aussi qu'un trop grand nombre de projets de tout genre au sein des écoles peut être nuisible à une démarche partenariale. Par exemple, dans l'une des écoles étudiées par ces auteurs, l'existence d'au moins sept ou huit partenariats entre des acteurs différents a contribué à augmenter le cynisme des enseignants face à la tentative de changement que cherchait pourtant à insuffler la direction d'école à travers les partenariats. Dans ce même contexte, un autre désavantage a émergé : l'esprit de consommation a affecté la participation des élèves qui se sont orientés davantage vers les projets de partenariat qui se déroulaient pendant l'été et qui les rémunéraient.

Les partenariats initiés par des acteurs externes à l'école tels que, par exemple, des dirigeants d'entreprises, entraînent eux aussi des difficultés particulières, prévient Sanders (2003). Ce type d'approche risque de déposséder les acteurs scolaires de toute initiative et de faire en sorte qu'ils ressentent que leur autorité est usurpée. Il est donc préférable que le personnel scolaire, les parents et les élèves co-participent à la planification et au développement du partenariat.

Les partenariats développés au niveau local permettent de mieux répondre aux besoins et de mettre en valeur les compétences au sein des milieux (Schweisfurth, 2005). Cependant, même si un projet de partenariat développé au niveau local peut connaître un franc succès, il peut aussi rencontrer divers obstacles lorsqu'il est reconduit à plus grande échelle. Par exemple, une entreprise partenariale ayant abouti à la création des écoles communautaires au Brésil, lesquelles offrent une scolarité jusqu'à la quatrième année du primaire et permettent une meilleure insertion des élèves afro-brésiliens, ne semble pas avoir de prise au-delà de la quatrième année, moment où les élèves doivent alors intégrer le système scolaire formel de l'État, un système qui a historiquement exclu et marginalisé les enfants afro-brésiliens (De Almeida, 2003).

Les partenaires ont parfois des buts, des priorités et des modes de fonctionnement divergents, ce qui peut circonvenir aux retombées ou gêner le bon déroulement d'un projet (Edens et Gilsinan, 2005). Les politiques internes divergentes de l'école et de l'organisme partenaire compliquent parfois le projet. Les buts des uns n'étant pas toujours en adéquation ou en complémentarité avec ceux des autres, des conflits peuvent survenir entre partenaires (De Almeida, 2003).

Des problèmes de communication surgissent également entre partenaires. Les référents culturels trop éloignés des partenaires peuvent parfois contrarier la bonne marche d'un projet (Schweisfurth, 2005). Des problèmes de rivalité peuvent aussi s'installer entre les partenaires et acteurs (Sanders, 2003). Les différences dans les standards de certification professionnelle, la comptabilité et la vérification des comptes, les sources de financement, les exigences de confidentialité et les restrictions à l'éligibilité représentent autant d'obstacles potentiels à la collaboration entre partenaires. Sanders (2003) suggère qu'une formation soit offerte aux partenaires afin de les habiliter à mieux collaborer entre eux. De même, le manque de diffusion de l'information au sujet du projet partenarial de l'école peut aussi nuire à son fonctionnement.

Ball (2007) constate la dissémination d'une culture de compétition dans le système d'éducation, une compétition se faisant sentir dans les relations entre partenaires ainsi que dans l'orientation des institutions vers une logique économique d'interdépendance liée aux avantages de la collaboration face à un désengagement financier de 
l'État dans ses institutions. Le partenariat devient un outil de cette collaboration à laquelle les établissements scolaires sont de plus en plus encouragés à adhérer. Cet auteur constate que le terme partenariat est ainsi devenu un mot à la mode, un moyen pour les écoles de s'assurer des ressources. Le terme est utilisé dans les discours et les politiques, et sert parfois à masquer les différences entre les secteurs public, privé et non lucratif, notamment en ce qui a trait aux modes de fonctionnement. De plus, Ball (2007) met à jour les relations de pouvoir inégales entretenues dans le cadre de projets où les partenaires, en théorie égaux, ont des objectifs différents, voire concurrents, et où peuvent dominer ceux d'un partenaire plus influent. Bref, les difficultés de fonctionnement, de gestion, de communication et de diffusion constituent des défis auxquels les acteurs des partenariats école-communauté sont appelés à faire face. De surcroît, la logique de marché dans laquelle s'inscrivent les partenariats peut engendrer des relations de pouvoir inégales entre les partenaires.

Ainsi, si l'on regroupe les éléments constitutifs mis en évidence ci-dessus en vue de construire une définition idéaltypique, il apparaît que :

le partenariat renvoie à une forme de coopération entre deux ou plusieurs organisations concourant à réaliser un projet qui réponde à une situation jugée problématique. Le projet partenarial est pensé ou initié par des acteurs locaux et implique la mise en commun de moyens matériels, intellectuels, humains ou financiers. Les partenaires ou organisations partenaires provenant équitablement de différentes sphères ou secteurs d'activités possèdent une connaissance sociopolitique du milieu dans lequel ils agissent. Les partenariats représentent des regroupements stables, lesquels possèdent des modes de fonctionnement, des structures et des échéanciers bien définis. Les partenaires tiennent des rôles complémentaires et égalitaires (collaboration), dégagent une compréhension mutuelle de l'objectif de leur partenariat, s'entendent sur les moyens de communication et de diffusion les plus adéquats et inclusifs, participent tous aux processus évaluatif et décisionnel, et bénéficient d'avantages mutuels grâce au partenariat.

Les multiples dimensions ou éléments qui se dégagent de cette définition idéaltypique fournissent un cadre conceptuel utile pour guider l'analyse des partenariats entre écoles et communautés. En particulier, pour les besoins de cette étude, nous retenons les éléments suivants

1. l'identification et la provenance des acteurs ou des partenaires, qui tiennent compte de l'importance de retrouver, dans toute entreprise partenariale, des acteurs autant du secteur scolaire que du secteur communautaire;

2. l'initiative du projet partenarial, qui renvoie à l'implication des partenaires scolaires et communautaires locaux dans la formulation d'objectifs communs;

3. la durée du projet, qui témoigne de la stabilité de l'entreprise partenariale permettant l'atteinte des objectifs à l'intérieur de limites de temps raisonnables;

4. les rôles dévolus aux partenaires, qui rendent compte du soin apporté à la définition et à la répartition équitable des rôles et des responsabilités de chacun.

\section{Position du problème et questions spécifiques de recherche}

La présente étude émerge d'une recherche plus vaste (Bélanger, Dalley, Dionne et Beaulieu, 2007a) s'intitulant : «Caractéristiques, composantes et modalités de mise en œuvre de projets scolaires-communautaires 
pour les écoles de langue française » (ÉCO). Cette étude visait à recenser et à décrire les projets de partenariat qui existent entre les écoles et les communautés francophones de l'Ontario et à créer un prototype pour guider d'éventuels partenaires intéressés à concevoir et à réaliser un partenariat (Bélanger, Dalley, Dionne et Beaulieu, 2007b). La multiplicité des partenariats recensés nous ont amenés à nous interroger sur la signification que les acteurs scolaires accordent à ces derniers. Ainsi, après avoir construit une définition idéaltypique de l'entreprise partenariale, nous cherchons à répondre plus spécifiquement aux questions suivantes :

1) Quel est le portrait des partenariats qui lient les écoles de langue française en Ontario et leur communauté?

2) Quelle est la signification que donnent les acteurs à la notion de partenariat? Quelle est leur compréhension de l'entreprise partenariale?

3) Les projets de partenariat déclarés correspondent-ils de près ou de loin à la définition idéaltypique d'un partenariat école-communauté telle que mise en évidence à partir de la recension des écrits, ou règne-t-il une certaine confusion entre partenariat, levée de fonds, projet ponctuel, activité de réseautage, etc.?

\section{Approche méthodologique}

\section{Participants}

Pour dresser un portrait des projets de partenariats en cours ou en voie de l'être dans les écoles de langue française de l'Ontario, nous avons sollicité la participation des douze (12) directions des conseils scolaires de districts de langue française et des deux (2) écoles séparées de langue française de l'Ontario. Nous avons obtenu le consentement de dix (10) de ces directions pour procéder au sondage dans les écoles de leur juridiction. Parmi ces directions, neuf (9) ont également accepté de répondre à un sondage téléphonique. Nous avons ensuite contacté les quelques 400 écoles potentiellement participantes et avons obtenu le consentement de 105 directions d'école ou responsables de partenariats dans ces écoles. Sur les 105 écoles ayant volontairement accepté de participer, $72 \%$ sont des écoles élémentaires et $28 \%$, des écoles secondaires; $60 \%$ sont des écoles catholiques et $40 \%$ sont publiques. Nous avons en plus catégorisé les écoles selon leur emplacement géographique : $40 \%$ d'entre elles sont situées dans ou à proximité de grands centres urbains et $60 \%$, à l'extérieur des grands centres. Les écoles participantes sont donc représentatives du milieu scolaire-communautaire de langue française en Ontario.

\section{Procédures et instruments de collecte de données}

La collecte des données a été réalisée en 2006-2007 par sondage téléphonique et par questionnaire administré en majorité au téléphone et en minorité par voie électronique. Le sondage téléphonique s'adressait aux directions des districts scolaires et des écoles séparées. II cherchait à identifier des projets de partenariats en cours dans les écoles des divers conseils et à recueillir des commentaires au sujet de ces projets.

Pour sa part, le questionnaire a été administré aux directions d'écoles ou aux responsables de partenariats dans les écoles (Voir questionnaire en annexe 1). Ce questionnaire visait, entre autres, à fournir des informations sur la nature et les objectifs des partenariats (questions 2a et 2b), l'initiative du projet (question 2c), la durée et l'avancée du projet (question 3 et 7), l'identification et la provenance des partenaires (question 5), et les rôles des partenaires (question 6). 


\section{Méthodes d'analyse}

En s'inspirant de la typologie de Sanders (2003), les partenariats déclarés par les acteurs scolaires ont été classés en fonction de leurs objectifs. Chaque questionnaire a été analysé en accordant une attention particulière aux questions portant sur le titre et les objectifs du partenariat déclaré. Les partenariats ont ainsi été répartis en trois grandes catégories axées soit sur les élèves, soit sur l'école, soit sur la famille. Les 105 questionnaires ont ainsi été comptabilisés. Alors que certains questionnaires portent sur plus d'un projet, chaque questionnaire a été placé dans une seule catégorie en fonction du projet prédominant.

Nous avons ensuite réalisé une analyse descriptive des réponses du sondage aux directions des conseils scolaires et des questionnaires aux acteurs scolaires. La méthode utilisée renvoie au calcul des occurrences des thèmes énoncés par les participants. L'analyse des données issues des sondages et des questions $2 \mathrm{a}$ et $2 \mathrm{~b}$ du questionnaire administré aux directions d'école permet de dresser un portrait des partenariats qui ont cours dans les communautés, tandis que l'analyse des questions 3, 5, 6 et 7 permet de cerner la signification que donnent les acteurs à la notion de partenariat en situant les partenariats recensés au regard des quatre (4) dimensions de la définition idéaltypique retenues. Ces diverses analyses permettent enfin de répondre à la troisième question de notre recherche, à savoir si les partenariats recensés se rapprochent ou non de la définition idéaltypique.

Signalons que par souci de confidentialité et afin de conserver l'anonymat des sujets, les titres des partenariats ont été modifiés, les personnes interrogées ont été identifiées par leur occupation (directeur, enseignant, agente de développement communautaire, etc.) et un code a été assigné aux sondages (S1, S5, etc.) et aux questionnaires (A1, A2, etc.).

\section{Analyse des résultats}

\section{Les partenariats école-communauté selon les directions des conseils scolaires}

Selon les directions des conseils scolaires interrogées, les partenariats école-communauté sont fréquents dans les écoles de langue française de l'Ontario. Quatre répondants en particulier insistent sur le fait qu'il existe de nombreux partenariats dans les écoles de leur conseil et élaborent sur les caractéristiques de ces partenariats.

Une directrice soutient qu'il «n'y a pas d'activité sans que soit impliquée la communauté » (S1). Elle précise qu'il y aurait des partenariats dans tous les secteurs : artistique, culturel, sportif, économique, social, etc. Parmi ceuxci, certains sont formels, d'autres informels. Une autre directrice abonde dans le même sens en précisant que, dans son petit village de 900 habitants, « la communauté est impliquée tout le temps » (S6). Un autre répondant assure que les partenariats école-communauté font partie du plan du conseil scolaire et qu'ils s'inscrivent dans la mise en œuvre de la politique d'aménagement linguistique (S8). En accord avec cette politique (MÉO, 2004), certains répondants insistent sur la nécessité d'établir des liens entre les écoles et leur communauté en contexte minoritaire francophone, "Les partenariats école-communauté sont indispensables», soutient une directrice (S1). Celle-ci ajoute que l'isolement pousse les gens à vouloir briser ce sentiment en établissant des liens entre eux.

En réfléchissant aux caractéristiques d'un modèle de partenariat, une directrice affirme que « le modèle doit s'inscrire dans l'ouverture » (S1). Les écoles qui font l'expérience de partenariats réussis seraient « ouvertes », c'est-à- 
dire ouvertes d'esprit et disposées à changer (Adger et Locke, 2000; FCCF, 2006; Johns, 2003; Ouellette et Lebreton Forbes, 1995). Toutefois, selon une répondante : «Les partenariats sont complexes à bâtir » (S10). Les partenaires engagés doivent en profiter à part égale : «C'est la base d'un partenariat solide : la réciprocité » (S10). Ceci rejoint les propos de Bouillon et Gomez (2001) pour qui la réciprocité ou les partenariats à avantages mutuels constituent une caractéristique des partenariats école-communauté réussis. De plus, de renchérir cette directrice, pour assurer la viabilité des partenariats, les échanges de services doivent être continuels. Selon une autre direction, les petits projets sont à privilégier : « Des projets de petite envergure peuvent faire une grande différence » (S1). Cette même direction rappelle que les communautés francophones de l'Ontario sont très diversifiées. II faut donc tenir compte des réalités régionales dans la conception et la mise en œuvre de partenariats. II est nécessaire pour les partenaires d'être sensibles aux différences régionales.

Les partenariats école-communauté constituent des entreprises courantes au sein des conseils scolaires francophones interrogés. Mais à quoi ressemblent concrètement ces entreprises partenariales? L'analyse des questionnaires administrés aux acteurs scolaires portant sur 105 partenariats permet d'en brosser un portrait.

\section{Portrait des partenariats école-communauté dans les écoles de langue française del'Ontario}

Tel que le révèle la lecture du tableau 1 (voir annexe 2), une majorité de partenariats sont axés sur les élèves. En effet, presque les deux tiers des partenariats recensés, soit $65 \%$ d'entre eux, sont orientés vers les élèves, leurs " besoins ", leur réussite scolaire et globale. Ces partenariats ont été classés en six sous-catégories. Premièrement, $14 \%$ des partenariats cherchent, d'une part, à favoriser la réussite scolaire des élèves, notamment en s'inscrivant dans la poursuite des objectifs curriculaires et, d'autre part, à faciliter la transition vers le postsecondaire ou le marché du travail. II s'agit de partenariats portant sur la littéracie, la communication orale, l'intégration des sciences dans les autres matières, de même que l'éducation coopérative. Certains de ces partenariats sont en collaboration avec des institutions postsecondaires ou des organismes de développement économique tel que le partenariat d'éducation coopérative qui donne aux élèves « la possibilité de tenter un emploi » (A74). Deuxièmement, 13\% des partenariats favorisent l'engagement des élèves dans leur communauté par des projets de bénévolat et des initiatives philanthropiques. Les écoles forment des partenariats avec des centres pour personnes âgées, des centres de santé communautaires, des organismes de charité ou des paroisses afin de sensibiliser les élèves à diverses causes, de les éduquer à la citoyenneté et d'amasser des fonds. Par exemple, une initiative conjointe de la part d'une école élémentaire catholique et d'un centre de santé communautaire vise à venir en aide aux sans-abri de la communauté (A68). Troisièmement, $11 \%$ des partenariats ont pour objectif d'initier les élèves aux arts et à la culture. II s'agit de partenariats avec des associations et des centres culturels et des membres de la communauté, notamment des artistes, grâce auxquels les élèves ont accès à des ressources et participent à des activités artistiques et culturelles. Une école secondaire publique du Nord de l'Ontario entretient un partenariat avec une association autochtone afin de "sensibiliser les élèves à d'autres cultures » (A38). Une autre école secondaire reprend à chaque année un projet d'apprentissage artistique et culturel pour les élèves par lequel elle crée des liens avec divers organismes et artistes de la communauté (A49). Quatrièmement, 10 \% des partenariats s'intéressent au développement et au bien-être social des élèves tels que le partenariat qui favorise l'intégration des élèves de milieux défavorisés par des programmes après les heures de classes (A41) et celui qui cherche à contrer l'intimidation (A50). Cinquièmement, $10 \%$ des 
partenariats visent plus particulièrement le développement d'un sentiment d'appartenance à la francophonie chez les élèves et un engagement envers leur communauté. Ces projets poursuivent des objectifs tels qu'« agrandir l'espace francophone [des élèves] » (A15), « développer un sentiment d'identité communautaire chez les élèves [par la création d'] une vidéo au sujet de l'histoire de la communauté » (A58) et « développer l'engagement envers la francophonie [en travaillant] avec les artistes francophones de la région» (A83). Sixièmement, $8 \%$ des partenariats visent le développement physique des élèves et offre à ces derniers une éducation à la santé, par exemple au sujet de la bonne nutrition, de l'éducation à la sexualité et de l'effet nocif du tabac et des drogues. Ces initiatives se concentrent sur le bien-être physique des élèves : " assurer que les enfants soient bien nourris et vêtus chaque jour » (A56); « offrir des petits-déjeuners » (A57); « analyser les besoins chez les jeunes et organiser des activités en fonction de ces besoins » (A61). Elles encouragent l'adoption de comportements jugés sains chez les élèves tels que la pratique des sports (A28, A51). Les écoles sont ici en partenariat avec des centres, bureaux ou agences de santé (A34, A48, A51), des associations sportives (A28), des parents (A48, A56, A57), des policiers (A99) et d'autres organismes ou membres de la communauté.

Un peu plus du quart des partenariats recensés (28\%) misent sur les écoles. Ces partenariats procurent des avantages aux écoles notamment quant à la mise en valeur de celles-ci au sein de la communauté. II s'agit de partenariats visant l'aménagement de l'école : la rénovation ou l'embellissement de la cour d'école (A27, A31, A42, A54, A67), la construction, l'amélioration et la maximisation de l'utilisation des installations (A47, A62). Quelques répondants rapportent aussi des activités de promotion de l'école : carnaval/festival (A70, A73) et journées portes ouvertes (A104). Certains répondants expliquent clairement qu'il s'agit de partenariats visant à « assurer la visibilité de l'école dans la communauté » (A104). Six pourcent (6\%) des partenariats recensés engagent la petite enfance et surtout les centres préscolaires. Ces projets cherchent à « faciliter l'intégration à l'école en maternelle » (A16) et à familiariser les jeunes enfants avec l'école de langue française (A35). Un autre $5 \%$ des partenariats recensés constituent des levées de fonds pour l'école afin de financer des activités éducatives (A69) ou d'alléger les coûts d'un programme sport-études (A20).

Enfin, un petit nombre de partenariats (7\%) visent les familles ou les parents principalement. D'une part, $4 \%$ des partenariats ont pour objectif d'informer et d'offrir des ressources aux parents, en leur proposant des ateliers (A23) et des journées d'information (A11), de même qu'en rendant accessibles des centres de ressources (A5). D'autre part, $3 \%$ des partenariats encouragent la participation des parents à l'école grâce à des activités spéciales, telles que des « dîners spaghettis et barbecues » (A94), ou par le biais d'organismes visant à outiller les parents dans leur rôle de premier éducateur en les engageant activement « dans le fonctionnement de l'école et l'apprentissage des enfants » (A33).

La catégorisation qui précède offre une vue d'ensemble des partenariats déclarés par les répondants et évoque certaines questions quant aux conceptions des partenariats école-communauté selon les acteurs scolaires. Si les réponses aux questionnaires montrent que les écoles de langue française en Ontario entretiennent avec leur communauté des activités multiples et diversifiées pour le bénéfice des élèves, de l'école et des parents, il apparaît important de mieux saisir ce que les acteurs scolaires entendent par partenariat école-communauté. Dans ce sens, il 
convient d'approfondir l'analyse en examinant jusqu'à quel point les caractéristiques des activités partenariales déclarées correspondent à celles identifiées dans la définition idéaltypique mise en évidence auparavant.

\section{Analyse des caractéristiques des partenariats école-communauté recensés en Ontario}

Quelle signification les acteurs scolaires accordent-ils à la notion de partenariat? Les partenariats écolecommunauté déclarés se rapprochent-ils de la définition de partenariat énoncée auparavant? Nous avons analysé les réponses de chaque questionnaire en fonction des quatre éléments que nous avons retenus pour guider cette analyse : l'identification et la provenance des partenaires, l'initiative du partenariat, sa durée et les rôles dévolus aux partenaires.

\section{Identification et provenance des partenaires scolaires et communautaires}

Grâce à un examen des réponses aux questions portant sur le titre et les objectifs du projet et l'identification des partenaires (questions $2 a, 2 b, 5 a$ et $5 b$ du questionnaire), il est possible de se prononcer sur la composition de l'équipe partenariale et la provenance des partenaires, à savoir s'ils proviennent du milieu scolaire ou communautaire. Les partenaires que nous avons classés comme provenant du secteur scolaire sont : la direction, le secrétariat, les membres du personnel, la conciergerie, les enseignants, les élèves, les clubs intrascolaires, le conseil d'école (bien qu'il regroupe des parents), le conseil scolaire et le ministère de l'Éducation de l'Ontario. Les partenaires provenant du secteur communautaire sont : les organismes, les associations, la municipalité, les représentants communautaires, les clubs extrascolaires, les centres de la petite enfance et préscolaires, les garderies, les parents et les autres membres de la communauté.

En accord avec cette classification, notons d'abord que $94 \%$ des partenariats engagent au moins un partenaire scolaire et un partenaire communautaire, et que la majorité (77\%) des partenariats déclarés inclut deux partenaires scolaires ou plus et deux partenaires communautaires ou plus. La majorité des partenariats (plus des trois quarts) se rapprochent donc, par cette caractéristique, de la définition idéaltypique.

On note toutefois des conceptions quelque peu différentes dans l'esprit de près du tiers des répondants (31 \%) en ce qui a trait à la provenance des partenaires. D'un côté, ces conceptions concernent les partenaires communautaires. En effet, $5 \%$ des répondants ont décrit des partenariats école-communauté sans identifier leur partenaire communautaire. Lorsque les partenaires sont identifiés, les partenaires communautaires sont parfois placés du côté scolaire, ou encore les mêmes partenaires sont notés sous les deux entrées scolaire et communautaire. Ainsi, $10 \%$ des répondants ont placé les parents du côté scolaire alors que le questionnaire mentionnait les parents parmi les exemples de partenaires communautaires (A6, A13, A44, A36, A48, A1, A49, A84, A22, A31); deux pourcent (2\%) des répondants ont placé les parents du côté scolaire et du côté communautaire (A22, A31); un répondant a classé l'association des parents catholiques du côté scolaire (A26). Sont aussi rangés sous les entrées scolaire et communautaire «les gens de la communauté et la ville » (A70) et les services policiers (A24). Les partenaires communautaires que sont les garderies, les centres de la petite enfance ou préscolaires sont rangés du côté des partenaires scolaires par $2 \%$ des répondants (A16, A45). Enfin, un directeur identifie «la personne-ressource qui s'occupe des aspects technologiques » autant comme partenaire scolaire que communautaire (A58). 
D'un autre côté, certains partenaires scolaires sont catégorisés en tant que partenaires communautaires, ou faisant partie des deux catégories scolaire et communautaire. Le conseil d'école a été identifié comme partenaire communautaire par $6 \%$ des répondants (A24, A25, A30, A33, A58, A73) et comme partenaire autant scolaire que communautaire par $2 \%$ d'entre eux (A24, A30). Le conseil scolaire a été classé par $2 \%$ des répondants dans la catégorie communautaire (A87, A100). Cinq pourcent (5\%) des répondants ont classé les écoles qui font partie de leur projet de partenariat comme partenaires communautaires (A29, A49, A91, A102, A104). Les élèves sont catégorisés en tant que partenaires scolaires et communautaires par $2 \%$ des répondants (A31, A77) et en tant que partenaires communautaires par $1 \%$ des répondants (A94). Les enseignants, les éducateurs, la direction ou le personnel sont catégorisés comme partenaires scolaires et communautaires par $4 \%$ des répondants (A31, A77, A84, A86). Dans le cadre d'un partenariat visant l'organisation d'un spectacle regroupant les chorales d'écoles (A29), l'animateur culturel, les directions des quatre écoles et les responsables des chorales sont placés du côté communautaire. Un directeur inscrit « l'aide de services à l'élève de l'école et le centre [scolaire] » comme partenaires communautaires (A6). Pour un autre, «l'intervenante en milieu scolaire » est considérée comme partenaire communautaire (A50).

Ces catégorisations de la part de $31 \%$ des répondants témoignent d'une conception différente, selon les directions d'école, de l'identification des partenaires selon qu'ils proviennent du monde scolaire ou communautaire et d'une certaine prédominance à localiser les partenaires dans le milieu scolaire..

\section{Initiative du projet partenarial}

Afin de documenter l'initiative du partenariat, nous avons demandé aux directions d'école lequel des partenaires, scolaire ou communautaire, avait initié le projet (question $2 c$ du questionnaire).

L'initiative d'une majorité des partenariats recensés (53\%) provient du secteur scolaire, la grande majorité de ceux-ci étant initiés directement par l'école. Parmi ceux-ci, $8 \%$ des répondants précisent que leurs partenariats ont été initiés par la direction de l'école elle-même. Cinq pourcent (5\%) des répondants affirment que le conseil scolaire a pris l'initiative du projet, un autre précise qu'il s'agit d'une initiative conjointe entre l'école et le conseil scolaire et enfin, un dernier attribue l'initiative de son partenariat au ministère de l'Éducation.

Par ailleurs, $16 \%$ des partenariats ont été entrepris par un ou plusieurs membres du secteur communautaire et $27 \%$ des partenariats ont été conjointement initiés par les secteurs scolaire et communautaire. Parmi ces derniers se trouve un partenariat qui n'a vu le jour que lorsque le conseil scolaire s'est approprié le projet : "L'idée avait déjà été lancée par l'école et la communauté, mais le projet n'avait pas eu lieu en raison d'une confusion en relation à la subvention. Le conseil scolaire a donc repris l'idée cette année et ils ont obtenu la subvention demandée » (A41). Enfin, deux (2) directions d'école n'ont pu retracer les acteurs à l'origine du partenariat et un répondant n'a pas fourni de réponse.

En somme, il apparaît que les partenariats écoles-communauté auxquels participent les écoles de langue française en Ontario sont plus souvent amorcés par le secteur scolaire, notamment par les écoles elles-mêmes, et misent sur la collaboration d'acteurs locaux. Cependant, il est permis de se demander jusqu'à quel point ces écoles impliquent les partenaires communautaires dans la détermination d'objectifs communs. Par contre, le $27 \%$ des écoles 
qui déclare une initiative conjointe école-communauté de leur partenariat se rapprochent davantage de la définition idéaltypique.

\section{Durée du projet partenarial}

Nous avons analysé les réponses recueillies au sujet de la durée et de l'état d'avancement du projet (questions 3 et 7 du questionnaire) afin de déterminer si les partenariats étaient d'une durée limitée et s'ils étaient soumis à un échéancier précis, puisque ces informations, selon la recension des écrits, représentent des indices de stabilité du partenariat.

La majorité des partenariats, soit $53 \%$ d'entre eux, sont d'une durée limitée ou suivent un calendrier précis. Par exemple, l'«Escale » représente un partenariat d'une durée de deux ans entre une école et une association culturelle, et vise à « favoriser l'intégration des élèves qui habitent dans un milieu défavorisé en leur offrant des programmes positifs après les heures de classe » (A41). Consciente de l'importance des étapes progressives de la démarche partenariale, la directrice interrogée précise qu'«il faut à présent roder le programme » (A41). Un échéancier précis serait un gage de réussite pour ces partenariats.

Par ailleurs, $32 \%$ des partenariats ne sont pas déterminés dans le temps. Il s'agit le plus souvent de projets informels échelonnés tout au long de l'année scolaire et de durée variable. Une école élémentaire catholique déclare entretenir plusieurs petits projets ponctuels tels que des levées de fonds et des collectes d'aliments pour diverses causes avec l'intention d'inciter les élèves à s'engager dans leur communauté (A52). Une autre école fait appel à des organismes communautaires afin de recueillir des fonds et des vêtements pour venir en aide aux « enfants dans le besoin » par le biais d'activités ponctuelles (A2). Treize pourcent (13\%) des répondants n'entrevoient pas la fin de leur projet. Tel est le cas d'un projet de création d'une spécialisation des métiers des arts de la scène mis sur pied en septembre 2006, afin de créer des liens dans le domaine des arts. Aussi, les partenariats visant l'engagement des élèves dans leur communauté, dans les secteurs de la santé (A13) ou économique (A12), à titre d'exemples, constituent des projets continus reconduits à chaque année. Ces projets indéterminés dans le temps apparaissent davantage, si l'on se rapporte à Newman (2001), comme des réseaux plutôt que des partenariats.

\section{Rôles au sein du partenariat}

La question sur les rôles des partenaires renseigne en partie sur les modes de fonctionnement et les structures du partenariat (question 6 du questionnaire) et permet de vérifier si les partenariats déclarés se rapprochent, selon cette caractéristique, de la définition idéaltypique. Plus précisément, les rôles sont-ils définis de façon claire? Sont-ils équitablement partagés entre les partenaires scolaires et communautaires?

D'abord, une majorité de répondants (71 \%), affirme qu'il y a bel et bien des rôles au sein de leur partenariat. Parmi ceux-là, 11 \% ne précisent pas si les rôles sont remplis par le secteur scolaire ou le secteur communautaire. Par exemple, dans le cadre d'un projet visant la mise en valeur de l'école au sein de la communauté, la directrice répond qu'il y a des rôles sans toutefois préciser qui les remplit : "Oui : trésorier, président, vice-président, secrétaire, membres du comité » (A54). Dans un autre cas, le partenariat qu'une école secondaire entretient avec un organisme francophone en vue d'organiser des activités culturelles en français dispose d'« un comité qui détermine qui joue tel rôle et quelles sont les tâches particulières à une activité » (A105). 
Par contre, $36 \%$ des répondants notent des rôles autant pour les partenaires scolaires que communautaires. Parlant d'un partenariat avec un institut postsecondaire, le directeur d'une école secondaire décrit le partage des rôles comme suit: "Le directeur a le rôle de continuer le projet, rôle d'administration, créer un lien avec le collège, l'enseignant de technologie à l'école enseigne la partie théorique, l'enseignant de technologie au Collège enseigne la partie pratique » (A90). Un autre $19 \%$ des répondants ont uniquement précisé les rôles des partenaires du secteur scolaire. Ainsi, un partenariat avec un organisme qui vient en aide aux jeunes ayant un handicap physique et dans le cadre duquel se tient une levée de fonds, attribue un rôle prépondérant aux enseignants : « Les enseignants organisent l'événement en divisant les classes et en organisant la compétition entre les classes de l'école » (A76). Parmi ces $19 \%$, deux répondants précisent que les rôles assurés par les partenaires scolaires sont remplis par la direction de l'école. Six pourcent $(6 \%)$ des répondants n'ont noté que les rôles assumés par le secteur communautaire. C'est le cas pour un partenariat entre une école primaire et un centre de la petite enfance visant à développer l'empathie chez les élèves; ce partenariat accorde le rôle d'animation d'ateliers dans les classes au partenaire communautaire, à savoir le centre de la petite enfance (A32). Enfin, $21 \%$ des répondants déclarent qu'il n'y a pas de rôle au sein de leur partenariat. Cinq d'entre eux précisent que les tâches sont plutôt partagées entre tous les partenaires, alors qu'un autre affirme qu'il ne voit «pas ça comme du partenariat» (A2). Sept pourcent (7 \%) des répondants n'ont pas fourni de réponse à la question.

En somme, un nombre relativement important de partenariats (36\%) fait état de la contribution des partenaires scolaires et communautaires dans la bonne marche du projet. Parmi les $25 \%$ qui n'indiquent qu'une contribution d'un secteur ou de l'autre en matière de rôles, il s'avère que les rôles dévolus au secteur scolaire apparaissent plus nombreux. Dans 71 \% des partenariats, les rôles des acteurs scolaires et communautaires sont définis. Les rôles ne sont cependant pas définis dans $29 \%$ des partenariats.

\section{Les partenariats école-communauté en Ontario : des partenariats qui se rapprochent ou qui s'éloignent de la définition idéaltypique des partenariats?}

Parmi les 105 partenariats déclarés, seulement $11 \%$ se rapprochent de la définition idéaltypique d'un partenariat école-communauté telle que construite dans cette étude en fonction des quatre dimensions analysées.

En termes d'identification et de provenance, ces partenariats regroupent des partenaires bien identifiés provenant des secteurs scolaire et communautaire. C'est le cas, par exemple, pour le partenariat «Santé et sport» (A51) qui vise à promouvoir l'activité physique, la saine alimentation et l'adoption de comportements sains chez les élèves de milieu défavorisé d'une école secondaire. Du côté scolaire, ce partenariat regroupe des acteurs tels que des élèves, des éducateurs et des enseignants. Du côté communautaire, on note l'implication d'un centre de santé communautaire et d'un réseau de développement économique. Dans un autre projet qui vise le développement de la «construction identitaire » (A15) et la communication orale des élèves de $7 \mathrm{e}$ et $8 \mathrm{e}$ années, notamment par un engagement dans la communauté (bénévolat, par exemple), la direction de l'école mentionne l'implication, du côté scolaire, des membres du personnel, de la direction, des élèves, de la coordonnatrice de l'aménagement linguistique et de la conseillère pédagogique attitrée au secondaire. Du côté communautaire, les acteurs incluent le comité du regroupement communautaire, les parents et des « invités dans la communauté ». 
En ce qui a trait à l'initiative, un projet d'aménagement de la cour d'école pour accommoder les enfants d'une garderie (A78) est le fruit d'une initiative partagée entre les secteurs scolaire et communautaire. II s'agit plus précisément d'acquérir de nouvelles structures de jeux et de les agencer en vue d'assurer la sécurité des enfants de l'école et de la garderie. Le directeur précise : «il faut s'assurer que les objectifs des deux partenaires soient communs et il faut bien y répondre [...] l'union fait la force » (A78). Cependant, même si l'initiative n'est pas décrite comme étant conjointe, des partenariats initiés autant par le secteur communautaire, tel le projet « Santé et sport » (A51) que par le secteur scolaire, tel que le projet « mini-apprentis » qui vise à accueillir les enfants de la garderie pour les familiariser avec l'école (A35), ont des objectifs auxquels les acteurs des deux secteurs peuvent se rallier.

La durée des projets partenariaux se révèle très variable. Par exemple, le projet d'aménagement de la cour d'école (A78) est d'une durée prédéterminée d'un an. Le projet "Santé et sport » (A51) se déroule selon un échéancier préétabli s'étalant sur une période de deux années scolaires. Pour sa part, le projet « mini-apprentis » qui vise à accueillir les enfants de la garderie pour les familiariser avec l'école (A35) se poursuit d'une année à l'autre, alors que le projet de « construction identitaire » (A15) se déroule pendant un mois et peut être renouvelé d'une année à l'autre. La durée de ces projets apparaît donc déterminée par la nature même des objectifs à atteindre.

Enfin, on note une attention particulière prêtée à la répartition équitable des rôles dans des projets tels que « mini-apprentis » (A35) où, selon la directrice de l'école « La directrice de la garderie avise les parents, les employés de la garderie accompagnent les petits en marchant et participent aux sessions animées ». Dans le projet «Santé et sport » (A51), une charte assigne à chacun des tâches bien précises. Dans le projet d'aménagement de la cour d'école (A78), les rôles de conscientisation auprès de la communauté et la recherche de fonds sont partagés entre les partenaires scolaires et communautaires. Le projet de regroupement communautaire (A14) qui vise à établir un partenariat solide entre l'école et la communauté engage une panoplie de partenaires communautaires. Des groupes ont été créés avec «le désir de collaborer et de se concerter pour assurer la viabilité de la francophonie », explique la répondante. Elle précise aussi que « chacun partage ce qui se passe dans son groupe membre et nous explorons des façons de s'appuyer et s'entraider les uns les autres ». Le partenariat entre une école élémentaire et des membres de la communauté, des organismes, des parents et trois paroisses cherche à voir au bien-être physique et social des élèves (A57). II se déroule tout au long de l'année scolaire et assigne des rôles bien définis aux divers acteurs notamment par la constitution de comités d'organisation. La directrice exerce un rôle de gestion, une personne ressource représente chaque organisme et un membre du clergé offre son soutien.

Par contre, plusieurs projets déclarés et recensés s'éloignent de la définition idéaltypique d'un partenariat école-communauté. Certains «partenariats » constituent plutôt des initiatives ponctuelles de l'école, notamment en vue de sa promotion dans la communauté. Ainsi, une école élémentaire du Nord de l'Ontario a tenu un «carnaval» (A91) afin de promouvoir l'établissement scolaire, mais n'a point réussi à susciter la participation de la communauté malgré la publicité faite par l'école. II s'agissait d'une initiative ponctuelle, sans engagement de partenaire communautaire, dans laquelle les rôles d'éventuels partenaires n'étaient pas définis.

Dans d'autres cas, il s'agit de projets visant à apporter des bénéfices pour l'école et les élèves. Lorsque questionné sur les partenariats école-communauté en cours dans son école, un directeur adjoint décrit des projets visant à recueillir des fonds et à offrir des expériences éducatives aux élèves. II s'agit d'initiatives de l'école, 
indéterminées dans le temps, qui visent à assurer des services et des ressources à l'école, sans qu'il n'y ait pour autant de retombées dans la communauté. Le directeur estime qu'il s'agit d'un « bon exemple de l'enrichissement que la communauté peut apporter à l'école » (A69). Dans le même sens, une directrice d'école décrit comme partenariats des activités occasionnelles de levées de fonds qui ne suivent pas de calendrier précis et sont organisées par le conseil d'école. Les rôles des divers partenaires ne sont pas précisés (A94). Une autre directrice décrit les projets de partenariat en cours dans son école comme n'étant « rien de formel, mais [impliquant] plusieurs organismes, ex. notre centre culturel, notre paroisse... » et ayant pour objectif l'« amélioration des services et expériences rendus à l'élève » (A25). Ces projets de durée variable sont généralement initiés par la directrice elle-même, les rôles n'y sont point définis et la provenance des partenaires est confuse, le conseil d'école étant considéré comme un partenaire communautaire. Pour sa part, un directeur d'école élémentaire répond que «le conseil d'école organise des activités pour les élèves et les parents [afin d'] impliquer [ces derniers] dans les activités de l'école » tout au long de l'année scolaire (A4). L'organisation des activités est prise en charge par le conseil scolaire et aucun partenaire communautaire n'est mentionné. Ainsi, il semble s'agir davantage d'activités ponctuelles organisées par le conseil d'école que d'un partenariat école-communauté relevant d'une collaboration stable entre divers partenaires scolaires et communautaires remplissant des rôles définis. Une asymétrie, telle que le mentionne Ball (2007), semble caractériser ces initiatives. Certains répondants se montrent conscients du manque de réciprocité ou du caractère unidirectionnel de leur "partenariat». Un directeur ayant décrit plusieurs petits projets informels poursuivant des objectifs curriculaires avec une panoplie de partenaires communautaires estime qu'il ne s'agit pas de « réels » partenariats. II affirme que «le terme "partenariat" est mal employé puisqu'il s'agit de dons de temps, d'expertise, d'argent et de ressources à sens unique (la communauté scolaire reçoit beaucoup et donne très peu) » (A53).

L'analyse des 105 questionnaires à partir de la définition idéaltypique des partenariats école-communauté révèle que seulement $11 \%$ des partenariats déclarés tiennent compte des quatre éléments de la définition idéaltypique analysés dans cette étude. La tendance relevée chez plusieurs acteurs scolaires à initier des projets partenariaux ponctuels relativement peu formalisés et pour leur propre bénéfice risque de renforcer le cynisme des acteurs communautaires envers ce genre de projets et de réduire leur collaboration potentielle.

\section{Résumé et conclusion}

Suite à une enquête menée en 2006 et 2007 pour le compte du ministère de l'Éducation de l'Ontario, visant à recenser et à décrire les projets de partenariat qui existent entre des acteurs des écoles de langue française en Ontario et ceux du milieu communautaire et associatif, nous avons cherché à mieux comprendre la réalité complexe que recouvre cette pratique de plus en plus encouragée. Ainsi, dans le cadre de cette étude, nous apportons des éléments de réponse aux questions suivantes : 1) Quel est le portrait des partenariats qui lient les écoles de langue française en Ontario et leur communauté? 2) Quelle est la signification que donnent les acteurs à la notion de partenariat? Quelle est leur compréhension de l'entreprise partenariale? 3) Les projets de partenariat déclarés correspondent-ils de près ou de loin à la définition idéaltypique d'un partenariat école-communauté telle que mise en évidence dans les écrits sur le sujet, ou règne-t-il une certaine confusion entre partenariat, levée de fonds, projet ponctuel, activité de réseautage, etc.? À partir de données provenant de sondages aux dirigeants de conseils scolaires et de questionnaires aux directions d'écoles, nous avons pu dresser un portrait des partenariats déclarés par 105 
participants en fonction des bénéficiaires. Nous avons ensuite analysé ces partenariats en concentrant notre attention sur quatre dimensions incluses dans la définition idéaltypique: l'identification et la provenance des partenaires, l'initiative des projets, la durée et les rôles dévolus à chacun.

Ainsi, il apparaît d'abord que les écoles de langue française en Ontario entretiennent avec leur communauté des activités partenariales multiples et diversifiées, considérés par certains comme indispensables. Ces partenariats visent surtout le bénéfice des élèves et des écoles, avec un petit nombre (7\%) qui s'adresse aux familles et aux parents. $\mathrm{Si}$, dans la majorité des cas, les partenaires proviennent à la fois de l'école et de la communauté, il s'avère que les répondants en recensent davantage dans le monde scolaire. Les partenariats écoles-communauté auxquels participent les écoles de langue française en Ontario sont le plus souvent initiés par le secteur scolaire, notamment par les membres du personnel de l'école, voire par les directions d'écoles elles-mêmes. Cette prédominance des partenaires scolaires, tant dans l'amorce du projet que dans son déroulement, comporte un certain risque. Si les partenaires scolaires savent reconnaître les problèmes ou les enjeux prioritaires pour les élèves et l'école en général, qu'en est-il des problèmes et des enjeux dans la communauté? Entre autres, il est permis de s'interroger sur la confusion qui règne chez les acteurs interrogés, à savoir si les partenaires proviennent du monde scolaire ou communautaire. Est-ce le reflet d'une connaissance limitée des relations sociales et de pouvoir existant dans la communauté, comme le suggère Leistyna (2002)?

Dans une majorité de partenariats, la durée et les rôles des acteurs scolaires et communautaires sont définis et témoignent de la stabilité du projet. Un nombre considérable de partenariats (36\%) fait état de la contribution des partenaires provenant à la fois du secteur scolaire et du secteur communautaire dans la bonne marche du projet. Parmi ceux qui n'indiquent qu'une contribution d'un secteur ou de l'autre quant aux rôles, il s'avère que les rôles dévolus aux partenaires du secteur scolaire apparaissent plus nombreux. Ces partenariats semblent donc représenter des regroupements stables à l'intérieur desquels les rôles sont déterminés, complémentaires et partagés entre les acteurs scolaires et communautaires, comme le recommandent des auteurs tels qu'Adger et Locke 2000, Lewis, 1999, Rainville, 2001 et Sanders, 2003. Cependant, il demeure que 32\% des projets déclarés sont de durée indéfinie et que $29 \%$ n'ont pas de rôles précis pour les acteurs. On peut donc s'interroger sur la stabilité et l'efficacité de telles entreprises.

À partir de l'analyse combinée des quatre dimensions retenues dans cette étude, nous estimons à $11 \%$ le nombre de projets partenariaux qui se rapprochent de la définition idéaltypique construite dans le cadre de cette recherche. Une certaine confusion semble donc régner quant à la définition d'un partenariat école-communauté chez la grande majorité les acteurs scolaires interrogés. En particulier, certains projets déclarés correspondent davantage à des projets de réseautage ou de levée de fonds, ou encore à des projets plus ponctuels, tel un spectacle ou un carnaval. Tel que le mentionne Ball (2007), cette notion de partenariat dorénavant présente dans le contexte de l'État décentralisé et managérial, semble parfois faire l'objet d'un usage abusif, étant donné que plusieurs activités décrites correspondent plutôt à des activités de mise en réseau et de financement des écoles.

Enfin, mentionnons quelques limites à notre étude. L'objectif initial de cet article était de brosser un portrait des partenariats école-communauté en cours dans les écoles de langue française en Ontario. Cette visée, en apparence aisée à réaliser, s'est avérée plus ardue qu'anticipée. En effet, la richesse et la multiplicité des partenariats 
décrits dans les questionnaires nous ont menées à nous interroger sur ce que les acteurs scolaires considèrent comme des partenariats école-communauté et en quoi ceux-ci se rapprochent d'une définition idéaltypique de tels partenariats. Cependant, le choix des acteurs scolaires en tant que seule source de données peut constituer une limite à notre analyse. En effet, ceux-là ont peut-être été enclins à décrire les partenariats dont ils avaient pris eux-mêmes l'initiative et à l'intérieur desquels ils jouaient des rôles prédominants. Un questionnaire auprès de partenaires communautaires se serait avéré un bon complément d'enquête.

Il demeure que les résultats de notre enquête montrent la pertinence d'offrir des ressources documentaires ou un accompagnement judicieux aux acteurs scolaires et communautaires, leur permettant de se faire une conception claire des partenariats école-communauté 2 et ainsi offrir de meilleures chances de succès à leurs entreprises partenariales.

\section{Références}

Adger, C. T. et Locke, J. (2000). Broadening the base : School/community partnerships serving language minority students at risk, Educational Practice Report, 6.

Association canadienne d'éducation de langue française-ACELF (2006). SNF 2006 - L'école Sacré-Cœur gagne le concours Actifs et fiers! [En ligne]. Disponible le 11 décembre 2006 : http://www.acelf.ca/c/presse/communiques/communique.php?id=61

Ball, S. (2007). Education plc - Understanding private sector participation in public sector education. London; New York : Routledge.

Barbour, C. et Barbour, N. H. (2001). Families, schools, and communities : Building partnerships for educating children. Upper Saddle River, New Jersey : Prentice-Hall.

Bélanger, N., Dalley, P., Dionne, L. et Beaulieu, G. (soumis). Les partenariats école-communauté et le marché scolaire de langue française en Ontario : « ça permet de vendre son école ». Revue des sciences de l'éducation.

Bélanger, N., Dalley, P., Dionne, L. et Beaulieu, G. (2007a). Rapport de recherche : Caractéristiques, composantes et modalités de mise en œuvre de projets scolaires-communautaires pour les écoles de langue française. Ministère de l'Éducation de l'Ontario, Ottawa, Ontario.

Bélanger, N., Dalley, P., Dionne, L. et Beaulieu, G. (2007b). Document prototype : Caractéristiques, composantes et modalités de mise en œuvre de projets scolaires-communautaires pour les écoles de langue française. Ministère de l'Éducation de l'Ontario, Ottawa, Ontario.

Bouillion, L. M. et Gomez, L. M. (2001). Connecting school and community with science learning : Real world problems and school Community partnerships as contextual scaffolds. Journal of Research in Science Teaching, 38(8), 878-898.

Broccolichi, S. et van Zanten, A. (1997). Espaces de concurrence et circuits de scolarisation. Les Annales de la recherche urbaine, 75, 5-17.

Burke, M. A. et Picus, L. O. (2001). Developing community-empowered schools. Thousand Oaks, CA : Corwin Press.

Chen, M., Konantz, J. L., Rosenfeld, M. L. et Frost, C. (2000), Working with urban schools that serve predominantly minority students. New Directions for Community Colleges, 111, Fall, 73-82.

De Almeida, A. F. J. (2003). Unveiling the mirror : Afro-Brazilian identity and the emergence of a community school movement. Comparative Education Review, 47(1), 41-63.

Dubé, J.-P. (1999). Frondeurs et tyrans. Le processus vers l'inclusion : comment les communautés francophones et les gouvernements doivent laisser tomber les rôles de David et Goliath pour collaborer pleinement au développement de l'éducation française. [En ligne]. Disponible le 10 décembre 2006 : http://www.ccppci.ca/documents/dube.html

Edens, R. et Gilsinan, J. F. (2005). Rethinking school partnerships. Education and Urban Society, 37(2), 123-138.

\footnotetext{
${ }^{2}$ Un tel document a été conçu à la suite de notre rapport. II peut être consulté sur le site de la Chaire de recherche Éducation et francophonies de la Faculté d'éducation de l'Université d'Ottawa.
} 
Epstein, J. L. et Sanders, M. G. (2000). Connecting home, school, and community : New directions for social research. Dans M. T. Hallinan (Dir.) : Handbook of the Sociology of Education. New York, NY : Kluwer Academic/Plenum Publishers.

Evergreen (2008). [En ligne]. Disponible le 2 juin 2008 : http://www.evergreen.ca.

Farmer, D. et Labrie, N. (2008). Immigration et francophonie dans les écoles ontariennes : comment se structurent les rapports entre les institutions, les parents et le monde communautaire? Revue des sciences de l'éducation, 34 (2), 377-398.

Fédération culturelle canadienne-française-FCCF (2006). Rapport final - Projet Élargissement de la mobilisation dans le dossier Lien langue-culture-éducation, Ottawa, Ontario, [En ligne]. Disponible le 2 juin 2008 : http://www.fccf.ca/documents/574.pdf

Gérin-Lajoie, D. (1995). L'école secondaire de Pain Court : une étude de cas. Étude sur les écoles exemplaires, Toronto : Association canadienne d'éducation.

Gérin-Lajoie, D. (2000). Les partenariats entre l'école et la communauté en milieu francophone minoritaire. Actes du colloque pancanadien sur la recherche en éducation en milieu francophone minoritaire : Bilan et prospectives. Moncton, NB : Centre de recherche et de développement en éducation (CRDE).

Johns, S. (2003). Partners in the leadership dance: School vocational learning partnerships and community development, The Educational Forum, 67(4), 317-325.

Leistyna, P. (2002). Extending the possibilities of multicultural community partnerships in urban public schools, The Urban Review, 34(1), 1-23.

Lewis, A. C. (1999). Communities working for better schools. Chicago, IL: Cross City Campaign for Urban School Reform.

Ministère de l'Éducation de l'Ontario-MÉO (2004). L'aménagement linguistique. Une politique au service des écoles et de la communauté de langue française de l'Ontario. Toronto, Ontario : Imprimeur de la Reine pour l'Ontario.

Navarro, S. et Natalicio, D. S. (1999). Closing the achievement gap in El Paso : Collaboration for K-16 renewal. Phi Delta Kappan, 80(8), 597-601.

Newman, J. (2001). Modernising governance : New labour, policy and society. London : Sage.

Ouellette, J.-G. et LeBreton Forbes, D. (1995). Étude de cas : École secondaire Népisiguit, Étude sur les écoles exemplaires, Toronto : Association canadienne d'éducation.

Paglin, C. (1998). Communities That Care. Personable approach to problem solving brings a warm, small-town feeling to urban neighbourhoods, Northwest Education, 4(2), Winter, 42-45, 52.

Rainville, M. (2001). L'élève francophone au cœur de la communauté. Cahier de stratégies et d'outils à l'intention des écoles et des composantes communautaires en vie francophone, pour réaliser des projets d'intégration communautaire avec des élèves de la maternelle à la $12^{e}$ année. Edmonton : La Fédération des parents francophones de l'Alberta.

Sanders, M. G. (2003). Community involvement in schools : From concept to practice. Education and Urban Society, 35(2), 61-180.

Scales, P. C., Roehlkepartain, E. C., Neal, M., Kielsmeier, J. C. et Benson, P. L. (2006). Reducing academic achievement gaps : The role of community service and service-learning. Journal of Experiential Education, 29(1), 38-60.

Schweisfurth, M. (2005). Learning to live together : A review of UNESCO's associated schools projects network. International Review of Education, 51(2/3), 219-234.

Shatkin, G. et Gershberg, A. (2007). Empowering parents and building communities: The role of school-based councils in educational governance and accountability. Urban Education, 42(6), 582-615.

Thomson, P. (2006). Miners, diggers, ferals and show-men: School-community projects that affirm and unsettle identities and places? British Journal of Education, 27(1), 81-96.

Van Zanten, A. (1997). Anthropological study of community-schools relations. Dans T. Husen et T. Postlethwaite (Dir.) : International Encyclopaedia of Education. Research and Studies, Londres : Pergamon Press.

Warren, M. (2005). Communities and schools: A new view of urban education reform, Harvard Educational Review, 75(2), $133-173$.

Zay, D. (2005). Partenariat. Dans P. Champy et C. Étévé (Dir.) : Dictionnaire encyclopédique de l'éducation et de la formation. Paris, France : Retz, 702-705. 


\section{Annexe 1. Questionnaire}

Ce questionnaire a pour but d'identifier le ou les partenariats en cours ou en voie de l'être dans votre école. Nous vous remercions pour votre participation.

\section{Coordonnées de l'école}
a) Nom de la personne (responsable du projet) qui remplit ce questionnaire
b) Fonction dans l'école
c) Nom de l'école
d) Nom du conseil scolaire
e) Adresse complète de l'école
f) Téléphone
g) Télécopieur
h) Courriel
i) Niveaux enseignés dans l'école
j) Nombre et type (première année, multi âges, niveaux multiples, concentration sports...) de classes, nombre d'élèves dans l'école

\section{Coordonnées du projet de partenariat}
a) Titre du projet de partenariat :
b) Objectifs visés par le partenariat :
c) Qui en a pris l'initiative, le secteur communautaire ou votre école ?

3. Durée du projet
a) Début :
b) Fin :

4. Quelle est votre définition de la communauté ?

5. Identification des partenaires

a) Qui, dans l'école, est impliqué dans le partenariat (membres du personnel, classes, clubs, élèves, etc.) ?

b) Qui, sur le plan communautaire, est impliqué dans le partenariat (représentant, association, parent, entreprise, musée, centre de sciences, organisme, artistes, etc.) ?

6. Y a-t-il des rôles au sein du projet de partenariat ? Si oui, lesquels?

7. Avancée du projet :

À quelle étape du projet êtes-vous rendus actuellement? Expliquez brièvement.
a) Au début :
b) Au milieu :
c) À la fin :

8. Nommez un ou deux obstacles que vous avez dû surmonter dans votre partenariat (financement, ressources, disponibilités, etc.) ?

9. Avantages du partenariat

a) Nommez un ou deux avantages que vous avez remarqués dans votre partenariat :

b) Est-ce que vous pensez que votre projet de partenariat est bénéfique pour : 
(4 étant le niveau le plus satisfaisant)

- la réussite scolaire des élèves :

1- 2 - 3- 4

- le cheminement scolaire des élèves :

1- 2 - 3- 4

- le développement social et culturel des élèves :

1_ 2 - 3- 4

- la mise en valeur de l'école au sein de la communauté :

$1-2-3-4$

- la mise en valeur dans votre milieu de l'organisme communautaire participant :

1- 2 - 3- 4

- les relations avec la communauté :

1- $2-3-4$

- le recrutement des élèves à l'école :

1_ 2 - 3- 4 -

- la rétention des élèves à l'école :

1- 2 - 3- 4

- le climat de l'école :

$1-2-3-4$

- l'implication du personnel :

$1-2-3-\quad 4$

- Autre (préciser)

10. Si vous recommandiez un tel projet à une autre école, que devrait-elle modifier, sachant les réussites et les échecs que vous avez connus?

11. Ajoutez ici tout commentaire que vous jugez pertinent à propos de votre projet : 
Annexe 2 : Tableau 1 : Types de partenariats déclarés par catégories

\begin{tabular}{|c|c|c|c|}
\hline CATÉGORIES & QUESTIONNAIRES & NOMBRE & EXEMPLES \\
\hline 1. Pour les élèves & & 68 & \\
\hline $\begin{array}{l}1.1 \text { Réussite scolaire, } \\
\text { objectifs curriculaires, } \\
\text { transition vers le } \\
\text { postsecondaire et marché du } \\
\text { travail }\end{array}$ & $\begin{array}{l}\text { A6, A36, A37, A40, } \\
\text { A53, A60, A63, A72, } \\
\text { A74, A81, A86, A89, } \\
\text { A90, A92, A97 }\end{array}$ & 15 & $\begin{array}{l}\text { Projet littéracie pour « remonter le } \\
\text { rendement des élèves surtout en français, } \\
\text { lecture et mathématiques » (A72) } \\
\text { L'éducation coopérative pour « donner la } \\
\text { possibilité de tenter un emploi » (A74) }\end{array}$ \\
\hline $\begin{array}{l}1.2 \text { Engagement des élèves } \\
\text { dans leur communauté }\end{array}$ & $\begin{array}{l}\text { A9, A12, A13, A21, } \\
\text { A22, A25, A39, A52, } \\
\text { A59, A68, A75, A76, } \\
\text { A93, A103 }\end{array}$ & 14 & $\begin{array}{l}\text { Plusieurs petits projet (levées de fonds, } \\
\text { collectes d'aliments non-périssables) afin } \\
\text { d'« inciter les élèves à s'impliquer dans } \\
\text { leur communauté » (A52) } \\
\text { Partenariat avec le centre de santé } \\
\text { communautaire afin de « venir en aide aux } \\
\text { sans-abri de la communauté » (A68) }\end{array}$ \\
\hline $\begin{array}{l}1.3 \text { Initiation aux arts et à la } \\
\text { culture }\end{array}$ & $\begin{array}{l}\text { A10, A17, A18, A29, } \\
\text { A38, A49, A55, A77, } \\
\text { A85, A88, A105 }\end{array}$ & 11 & $\begin{array}{l}\text { Partenariat avec un centre culturel } \\
\text { afin « d'accroître la participation des } \\
\text { élèves à des activités culturelles » (A10) } \\
\text { Partenariat avec une association } \\
\text { autochtone afin de « sensibiliser les élèves } \\
\text { à d'autres cultures » (A38) }\end{array}$ \\
\hline $\begin{array}{l}\text { 1.4 Développement et bien- } \\
\text { être social des élèves }\end{array}$ & $\begin{array}{l}\text { A2, A19, A24, A32, } \\
\text { A41, A43, A44, A45, } \\
\text { A50, A65 }\end{array}$ & 10 & $\begin{array}{l}\text { Programme pour « réduire l'intimidation à } \\
\text { l'école » (A19) } \\
\text { «Favoriser l'intégration des élèves qui } \\
\text { habitent dans un milieu défavorisé en leur } \\
\text { offrant des programmes positifs après les } \\
\text { heures de classe » (A41) }\end{array}$ \\
\hline $\begin{array}{l}\text { 1.5 Développement d'un } \\
\text { sentiment d'appartenance à } \\
\text { la francophonie et à leur } \\
\text { communauté }\end{array}$ & $\begin{array}{l}\text { A1, A15, A46, A58, } \\
\text { A66, A80, A82, A83, } \\
\text { A96, A101 }\end{array}$ & 10 & $\begin{array}{l}\text { Projet de recherche-action visant à } \\
\text { "agrandir l'espace francophone » des } \\
\text { élèves (A15) } \\
\text { Partenariat avec une artiste francophone } \\
\text { sur la chanson et les légendes afin de } \\
\text { « développer l'engagement envers la } \\
\text { francophonie » (A83) }\end{array}$ \\
\hline $\begin{array}{l}\text { 1.6 Développement } \\
\text { physique et éducation à la } \\
\text { santé }\end{array}$ & $\begin{array}{l}\text { A28, A34, A48, A51, } \\
\text { A56, A57, A61, A99 }\end{array}$ & 8 & $\begin{array}{l}\text { Programmes anti-tabagisme et bonne } \\
\text { nutrition pour « réduire la consommation } \\
\text { de cigarettes » et « instaurer de bonnes } \\
\text { habitudes alimentaires chez nos élèves » } \\
\text { (A34) } \\
\text { Pratiques de soccer intérieur organisées } \\
\text { par l'association de soccer pour } \\
\text { « développer les habiletés au soccer » } \\
\text { (A28) }\end{array}$ \\
\hline
\end{tabular}




\begin{tabular}{|c|c|c|c|}
\hline 2. Pour l'école & & 29 & \\
\hline $\begin{array}{l}\text { 2.1 Mise en valeur de l'école } \\
\text { dans la communauté }\end{array}$ & $\begin{array}{l}\text { A7, A8, A14, A26, A27, } \\
\text { A31, A42, A47, A54, } \\
\text { A62, A67, A70, A73, } \\
\text { A78, A87, A91, A98, } \\
\text { A102, A104 }\end{array}$ & 19 & $\begin{array}{l}\text { Projet d'aménagement de la cour d'école } \\
\text { pour « avoir de nouvelles structures de } \\
\text { jeux » (A67) } \\
\text { Projets avec la bibliothèque municipale et } \\
\text { la paroisse afin de « donner de la visibilité } \\
\text { à l'école » (A8) }\end{array}$ \\
\hline $\begin{array}{l}2.2 \text { Centres de la petite } \\
\text { enfance }\end{array}$ & $\begin{array}{l}\text { A16, A35, A79, A84, } \\
\text { A95, A100 }\end{array}$ & 6 & $\begin{array}{l}\text { Partenariat afin d'« accueillir les } \\
\text { petits/petites de la garderie pour les } \\
\text { familiariser avec l'école » (A35) }\end{array}$ \\
\hline $\begin{array}{l}2.3 \text { Cueillette de fonds pour } \\
\text { l'école (voyages éducatifs, } \\
\text { programmes spéciaux, etc.) }\end{array}$ & A3, A20, A64, A69 & 4 & $\begin{array}{l}\text { Partenariat avec la municipalité pour } \\
\text { l'utilisation de l'aréna afin d'« alléger les } \\
\text { coûts du programme sport-études » (A20) } \\
\text { Projets pour « récolter des subventions et } \\
\text { offrir aux élèves des expériences } \\
\text { éducatives à l'extérieur des murs de } \\
\text { l'école » (A69) }\end{array}$ \\
\hline 3. Pour les familles/parents & & 7 & \\
\hline $\begin{array}{l}3.1 \text { Informer et offrir des } \\
\text { ressources aux parents }\end{array}$ & $A 5, A 11, A 23, A 30$ & 4 & $\begin{array}{l}\text { Centre de ressources pour les parents } \\
\text { pour « fournir aux parents francophones et } \\
\text { anglophones des outils pour favoriser le } \\
\text { bien-être, le rendement et l'apprentissage } \\
\text { de leurs enfants » (A5) }\end{array}$ \\
\hline $\begin{array}{l}3.2 \text { Encourager la } \\
\text { participation des parents à } \\
\text { l'école }\end{array}$ & A4, A33, A94 & 3 & $\begin{array}{l}\text { Activités organisées par le conseil d'école } \\
\text { afin d'« impliquer les parents dans les } \\
\text { activités de l'école » (A4) }\end{array}$ \\
\hline Aucun & A71 & 1 & \\
\hline Total & & 105 & \\
\hline
\end{tabular}

